\title{
Frecuencia de anticuerpos contra el virus de la hepatitis $E$ en donantes de sangre del municipio de Yarumal, Antioquia
}

\section{Frequency of Antibodies to Hepatitis E In Blood Donors in the Municipality of Yarumal, Antioquia}

Alejandra Duque Jaramillo, Biol. ${ }^{1}$, Luisa F. Restrepo, Ing. Biomédica'1, Carolina Mantilla-Rojas, Biol. ${ }^{1}$, Mónica Toro, Biol. ${ }^{1}$, Juan Camilo Olarte, Bact. Esp. ${ }^{2}$, W. Alfredo Ríos Ocampo, Biol. MSc1 ${ }^{1}$, Fabián Cortés-Mancera, Bact., MSc ${ }^{1,3}$,

María Cristina Navas, Bact., MSc, PhD'.

1 Grupo de Gastrohepatología, Facultad de Medicina, Universidad de Antioquia. Medellín, Colombia.

2 Banco de Sangre, Cruz Roja, Seccional Antioquia. Medellín, Colombia.

3 Grupo de Investigación e Innovación Biomédica GI2B, Facultad de Ciencias Exactas, Instituto Tecnológico Metropolitano. Medellín, Colombia.

Fecha recibido: $26-02-16$ Fecha aceptado: 25-07-16

\begin{abstract}
Resumen
Introducción: el virus de la Hepatitis E (VHE), transmitido por la ruta fecal-oral, causa enfermedad hepática aguda. En Colombia se han realizado algunos estudios en pacientes con diagnóstico de hepatitis viral, en trabajadores de fincas porcícolas, en población porcina y en muestras ambientales. Objetivo: evaluar la presencia de anticuerpos anti-VHE en muestras de donantes de sangre del municipio de Yarumal, departamento de Antioquia. Metodología: se obtuvieron muestras de suero de donantes de sangre colectadas por la Cruz Roja Colombiana en una campaña de donación voluntaria en el municipio de Yarumal. En las muestras se determinó la presencia de anticuerpos anti-VHE tipo lgM e lgG mediante estuche comercial de ELISA. Resultados: se analizaron 42 muestras de suero, 19 de las cuales $(45,2 \%)$ fueron positivas para anticuerpos anti-VHE IgG. Ninguna de las muestras fue positiva para anticuerpos anti-VHE tipo IgM. Conclusiones: este es el primer reporte de anticuerpos anti-VHE en donantes de sangre en Colombia. La frecuencia de anti-VHE $(45,2 \%)$ es mayor a lo reportado previamente en otros estudios realizados en el país y a lo reportado en donantes de sangre en otros países de América Latina. Esta frecuencia podría estar relacionada con el contacto con cerdos infectados, así como con la exposición a agua contaminada con el virus. Sin embargo, estudios adicionales deben ser realizados en otras poblaciones similares en el país para confirmar este hallazgo.
\end{abstract}

\section{Palabras clave}

Virus de la Hepatitis E, Anticuerpos, ELISA, donantes de sangre, población rural.

\begin{abstract}
Introduction: The hepatitis $\mathrm{E}$ virus (HEV) is transmitted via the fecal-oral route and causes acute liver disease. In Colombia there have been some studies of patients who have been diagnosed with viral hepatitis, of swine farm workers and in environmental samples. Objective: The objective of this study was evaluate samples from blood donors in the municipality of Yarumal in the department of Antioquia for the presence of anti-HEV antibodies. Methods: Serum samples were obtained from blood donated to the Colombian Red Cross by blood donors on a voluntary basis in a campaign in the municipality of Yarumal. Samples in the presence of anti-HEV IgM and IgG ELISA using commercial kit was determined.Results: Forty-two serum samples were analyzed: 19 (45.2\%) were positive for anti-HEV IgG. None of the samples were positive for anti-HEV IgM. Conclusions: This is the first report of anti-HEV antibodies in blood donors in Colombia. The frequency of anti-HEV (45.2\%) is higher than previously reported in other studies in this country and in blood donors in other Latin American countries. This frequency may be linked to contact with infected pigs and to exposure to water contaminated with the virus. However, additional studies should be conducted in similar populations in the country to confirm this finding.
\end{abstract}

\section{Keywords}

Hepatitis E virus, antibodies, ELISA, blood donors, rural populations. 


\section{INTRODUCCIÓN}

El Virus de la Hepatitis E (VHE) es un agente etiológico de hepatitis viral aguda de transmisión entérica. La Organización Mundial de la Salud estima que cada año hay 20 millones de infecciones por VHE, más de 3 millones de casos sintomáticos y 56600 muertes relacionadas con el virus (1). El VHE (Orthohepevirus A) pertenece a la familia Hepeviridae, género Orthohepevirus, que incluye virus que infectan mamíferos (humanos, cerdos, jabalíes, venados) y aves (2). Es un virus no envuelto, cuyo genoma de ARN de cadena simple y polaridad positiva de $7,3 \mathrm{~kb}$ contiene tres marcos de lectura abiertos (ORF, Open Reading Frame) y dos regiones no codificantes (5' y 3'-UTR, untranslated region) altamente conservadas (3).

Se han identificado cuatro genotipos del VHE con base en el análisis filogenético del ORF2 (4). Los genotipos 1 y 2 infectan exclusivamente humanos y han sido relacionados con epidemias en países en vía de desarrollo; por otro lado, los genotipos 3 y 4 infectan humanos, cerdos y otras especies animales, y se han reportado en casos esporádicos de infección, principalmente en países industrializados $(5,6)$. En América Latina se ha descrito la circulación de los genotipos 1 y 3 ; mientras que el genotipo 2 fue descrito en un único evento epidémico en México (7).

Su principal vía de transmisión es la ruta fecal-oral, a través del consumo de agua y alimentos contaminados con heces, aunque puede presentarse transmisión zoonótica por consumo de vísceras de cerdo mal cocidas, en particular hígado, y por exposición a heces de animales infectados (8). Otras rutas de transmisión menos frecuentes son la transfusión de componentes sanguíneos y la transmisión vertical $(9,10)$. Con respecto al diagnóstico de la infección por VHE, esta no presenta características clínicas que permitan distinguirla de otras hepatitis virales; por lo tanto, el diagnóstico se basa en la detección de anticuerpos antiVHE tipo IgM e IgG por ELISA, así como en la detección del genoma viral mediante RT-PCR (5).

Los primeros estudios de la infección por VHE en Colombia se realizaron en pacientes con diagnóstico de hepatitis viral, en los que se describieron marcadores serológicos y moleculares; además se identificó el genotipo 3 del VHE $(11,12)$.

Luego, estudios en trabajadores de fincas porcícolas demostraron la presencia de anticuerpos anti-VHE tipo $\operatorname{IgG}(13,14)$, mientras que en cerdos de granjas de producción se detectaron anticuerpos anti-VHE tipo IgM e IgG y el genoma viral en hígado, y se caracterizó el virus en muestras de heces (15-17).

Se han determinado 4 genotipos y 24 subtipos de VHE. La presencia de cepas virales tanto en humanos como en otras especies, ha sugerido que existen reservorios animales para VHE, entre los cuales el cerdo juega un papel importante.

Teniendo en cuenta que el departamento de Antioquia es el principal productor y consumidor de carne de cerdo en Colombia, se investigó la presencia de este virus en los cerdos de diferentes granjas porcícolas, con el fin de determinar anticuerpos anti-HEV y RNA viral en heces porcinas. Adicionalmente, se ha detectado el VHE genotipo 3 en aguas de abastecimiento y residuales en municipios de Antioquia (Zaragoza, San Pedro de los Milagros, Granada, Puerto Berrío, Frontino, Girardota, Venecia y Cisneros) (18). Sin embargo, no existen datos de la frecuencia de antiVHE en donantes de sangre en Colombia. En el presente estudio se propuso evaluar la presencia de anti-VHE en muestras de donantes de sangre del municipio de Yarumal, departamento de Antioquia.

\section{MATERIALES Y MÉTODOS}

\section{Tipo de estudio}

Descriptivo de corte transversal.

\section{Población de estudio}

El municipio de Yarumal está localizado en la subregión norte del departamento de Antioquia, a 120 kilómetros de Medellín, capital del departamento (figura 1); su cabecera está conformada por 27 barrios y la zona rural cuenta con 45 veredas y 7 corregimientos (19). De acuerdo con datos del Departamento Administrativo Nacional de Estadística (DANE), en el 2006 el municipio contaba con 31816 habitantes, de los cuales 26716 (83,9\%) residían en la cabecera municipal y $5100(16,02 \%)$ en la zona rural (20). Con respecto a la actividad económica, el $80 \%$ de las viviendas rurales tienen actividad agropecuaria y, de estas, el 95,2\%, actividad pecuaria (20). En el 2006, el 87,6\% de la población tenía acceso al servicio de acueducto y el $84,4 \%$ al servicio de alcantarillado (20). El municipio de Yarumal cuenta con planta de tratamiento de agua potable desde 2007 (21).

\section{Muestras}

Se evaluaron 50 muestras de suero de donantes de sangre del municipio de Yarumal, obtenidas por la Cruz Roja Colombiana durante el segundo semestre de 2012. Se obtuvo consentimiento por parte de todos los participantes en el estudio. El suero obtenido se congeló a $-20{ }^{\circ} \mathrm{C}$ hasta su análisis en el Laboratorio de Gastrohepatología de la Universidad de Antioquia. A partir del formulario de 


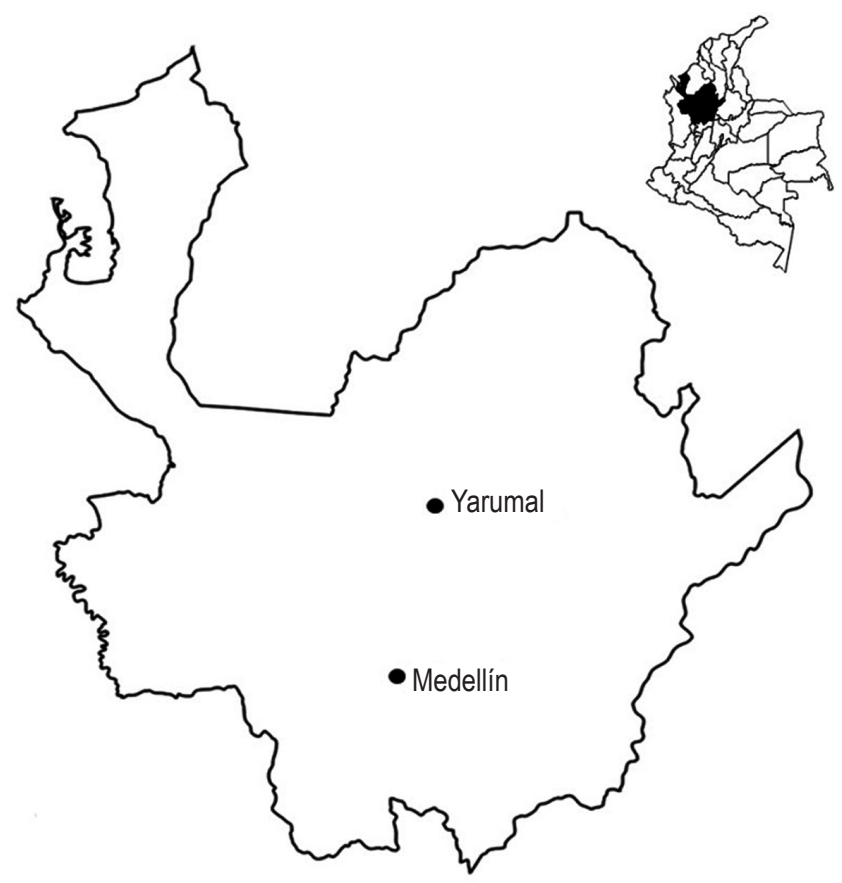

Figura 1. Ubicación del municipio de Yarumal y de la ciudad de Medellín en el departamento de Antioquia.

donación se obtuvieron datos de edad, género y ocupación. Este estudio fue aprobado por el comité de ética del Instituto Tecnológico Metropolitano (ITM).

\section{Detección de anticuerpos anti-VHE}

La presencia de anticuerpos anti-VHE tipo IgM e IgG en las muestras de suero fue evaluada utilizando estuches comerciales de ELISA (HEV Dia.pro, Diagnostic Bioprobes, Milán, Italia). Este estuche utiliza antígenos sintéticos VHE-específicos que codifican para los determinantes conservados e inmunodominantes derivados de cepas de México y Myanmar (22). La sensibilidad y especificidad del kit es del 99\% según el fabricante. De las 50 muestras de suero, 42 fueron evaluadas; las 8 muestras restantes no fueron incluidas en el análisis por volumen insuficiente. Las 42 muestras fueron analizadas por duplicado y los resultados analizados de acuerdo con las instrucciones del fabricante; así, se determinó la razón densidad óptica/punto de corte, y las muestras con resultado $>1,1$ se consideraron como positivas, entre 0,9 y 1,1 como indeterminadas, y $<0,9$ como negativas.

\section{RESULTADOS}

El rango de edad de los donantes fue de 17 a 58 años, con un promedio de 33,4 años. De las muestras analizadas, el 78,6\% provenía de mujeres y el $21,4 \%$ de hombres. La población fue dividida según su ocupación en estudiantes, amas de casa y empleados. La información disponible de los donantes no permite discriminar entre la población de la cabecera municipal y de las veredas del municipio. Diecinueve muestras de suero $(45,2 \%)$ fueron positivas para los anticuerpos anti-VHE tipo IgG, pero ninguna presentó anticuerpos anti-VHE tipo IgM. Las muestras con un resultado indeterminado se consideraron negativas. La seropositividad de acuerdo con el género, la edad y la ocupación se resume en la tabla 1 .

Tabla 1. Características sociodemográficas y seropositividad de anticuerpos anti-VHE IgG en una población de donantes de sangre del municipio de Yarumal, Antioquia.

\begin{tabular}{|c|c|c|c|c|c|c|}
\hline & \multicolumn{2}{|c|}{$\begin{array}{c}\text { Total } \\
(n=42)\end{array}$} & \multicolumn{2}{|c|}{$\begin{array}{c}\text { IgG VHE } \\
\text { positivo } \\
\text { (n=19, 45,2\%) }\end{array}$} & \multicolumn{2}{|c|}{$\begin{array}{c}\text { IgG VHE } \\
\text { negativo } \\
\text { (n= }=23,54,8 \%)\end{array}$} \\
\hline & $\mathrm{n}$ & $\%$ & $n$ & $\%$ & $\mathrm{n}$ & $\%$ \\
\hline \multicolumn{7}{|l|}{ Género } \\
\hline Masculino & 9 & $21,4 \%$ & 5 & $26,3 \%$ & 4 & $17,4 \%$ \\
\hline Femenino & 33 & $78,6 \%$ & 14 & $73,7 \%$ & 19 & $82,6 \%$ \\
\hline \multicolumn{7}{|l|}{ Edad } \\
\hline $17-20$ & 6 & $14,3 \%$ & 2 & $10,5 \%$ & 4 & $17,4 \%$ \\
\hline $20-39$ & 21 & $50,0 \%$ & 10 & $52,6 \%$ & 11 & $47,8 \%$ \\
\hline $40-59$ & 15 & $35,7 \%$ & 7 & $36,8 \%$ & 8 & $34,8 \%$ \\
\hline \multicolumn{7}{|l|}{ Ocupación } \\
\hline Estudiante & 12 & $28,6 \%$ & 5 & $26,3 \%$ & 7 & $30,4 \%$ \\
\hline Ama de casa & 12 & $28,6 \%$ & 5 & $26,3 \%$ & 7 & $30,4 \%$ \\
\hline Empleado & 18 & $42,9 \%$ & 9 & $47,4 \%$ & 9 & $39,1 \%$ \\
\hline
\end{tabular}

\section{DISCUSIÓN}

El presente reporte corresponde al primer estudio de VHE en donantes de sangre realizado en Colombia. Se analizaron muestras de suero de la población de Yarumal y se detectaron anticuerpos anti-VHE tipo IgG en el $45,2 \%$ de las muestras, pero no anticuerpos tipo IgM. Esta alta seroprevalencia puede estar relacionada con el contacto de las personas con cerdos infectados debido a que la ganadería porcina hace parte de la actividad económica del municipio de Yarumal (20), así como con la exposición a agua contaminada con el virus previo a la puesta en funcionamiento de la planta de tratamiento de agua potable en 2007 (21).

Las campañas de donación de sangre se realizan en el parque principal, generalmente entre las 9:00 a.m. y las 4:00 p.m., lo que puede explicar el alto porcentaje de donantes de sexo femenino, teniendo en cuenta que la mayoría declaró como ocupación ser estudiante o ama de casa; mientras que todos los hombres registraron ser empleados en diferentes oficios. 
Llama la atención que la seropositividad de anticuerpos anti-VHE tipo IgG encontrada en este estudio es notablemente superior a lo descrito en América Latina y en estudios previos realizados en Colombia. En muestras de pacientes con diagnóstico clínico de hepatitis virales provenientes de 15 de los 32 departamentos del país se describió una frecuencia de 6,3\% (16/253) para anti-VHE tipo IgG, mientras que en muestras de pacientes en Medellín la frecuencia de anticuerpos IgG fue de 10,9\% (10/91) (11). Por otro lado, en trabajadores de fincas porcícolas de Caldas, San Pedro y Guarne (Antioquia), Betancur y colaboradores reportaron una frecuencia de anticuerpos IgG del 11,2\% (11/98) (13), similar a la frecuencia de 15,7\% (25/159) IgG/IgM descrita por Gutiérrez y colaboradores en un estudio realizado en 10 municipios del departamento de Antioquia (Barbosa, Bello, Caldas, Medellín, Entrerríos, Don Matías, San Pedro, Santa Rosa de Osos, Jericó y Rionegro) en personas con exposición ocupacional a porcinos (14). Estas personas constituyen una población de riesgo para la infección por VHE; sin embargo, en todos los casos la seropositividad fue menor a la encontrada en este estudio.

Gutiérrez y colaboradores reportan, además, una seropositividad anti-VHE de 5,9\% (2/34) en individuos convivientes con personas con exposición laboral a cerdos, y de $7,2 \%$ (71/983) en población general de la zona urbana de dichos municipios (14). Un reporte reciente evaluó la coinfección por el virus de la Hepatitis E y otros agentes etiológicos de hepatitis viral en muestras de suero de pacientes de diferentes regiones del país, y encontró una frecuencia de anticuerpos anti-VHE tipo IgG del 25,3\% (342/1097), tipo IgM del 5,6\% (126/1097), y, de ambos marcadores, del $5,8 \%(64 / 1097)$ (23). Esta seroprevalencia de anticuerpos tipo IgG es la más cercana a la reportada en el presente estudio, pero las poblaciones no son comparables, pues se trata de individuos positivos para marcadores de infección por los virus de la Hepatitis A, de la Hepatitis B y de la Hepatitis $\mathrm{C}$, quienes tendrían mayor riesgo de estar infectados con el VHE. Es de anotar que la frecuencia de anticuerpos difiere de lo reportado previamente en individuos con diagnóstico clínico de hepatitis virales en el país (11).

En un estudio en la población rural de Durango, México, se describió una frecuencia de anti-VHE tipo IgG de 36,6\% $(100 / 273)$ y $40,7 \%(61 / 150)$, similar a la descrita en el presente estudio $(24,25)$. Mientras que en mujeres embarazadas y menonitas, en esta misma población, se reportaron frecuencias de anticuerpos anti-VHE del 5,7\% (25/439) y 6,7\% $(10 / 150)$ respectivamente $(25,26)$. Estudios en población rural de Bolivia $(27,28)$, Brasil (29-31) y Venezuela (30) reportan entre $3,9 \%$ y $12,9 \%$ de anti-VHE tipo IgG (tabla 2 ). Diferencias entre los factores de riesgo de las poblaciones rurales, tales como el acceso al agua potable, la infraestruc- tura del alcantarillado, actividades económicas y los hábitos de higiene, podrían explicar este rango de seroprevalencia. Se debe considerar también la sensibilidad y especificidad de las metodologías utilizadas. Adicionalmente, la mayoría de estos estudios no son recientes y podrían no reflejar la situación actual de las poblaciones.

Tabla 2. Seroprevalencia del VHE en población rural latinoamericana.

\begin{tabular}{lccccc}
\hline País & $\begin{array}{c}\text { Población } \\
\text { de estudio } \\
\mathrm{n}=\end{array}$ & $\begin{array}{c}\text { Rango } \\
\text { edad } \\
\text { (años) }\end{array}$ & $\begin{array}{c}\text { Positividad } \\
\text { anti-VHE } \\
\text { IgG }\end{array}$ & Año* & Ref. \\
\hline Bolivia & 490 & $1-85$ & $7,3 \%$ & 1997 & $(27)$ \\
& 236 & $1-87$ & $6,3 \%$ & 2006 & $(28)$ \\
Brasil & 145 & $\mathrm{ND}$ & $2,1 \%$ & $1996-1997$ & $(29)$ \\
& 388 & $<1-90$ & $12,9 \%$ & 2004 & $(30)$ \\
& 310 & $11-98$ & $8,4 \%$ & $2009-2010$ & $(31)$ \\
México & 273 & $18-91$ & $36,6 \%$ & $2006-2007$ & $(24)$ \\
& 150 & $18-91$ & $40,7 \%$ & $2006-2007$ & $(25)$ \\
Venezuela & 204 & $4-36$ & $3,9 \%$ & 1992 & $(32)$ \\
\hline
\end{tabular}

* Año en el que se recolectaron las muestras para el estudio. ND: información no disponible.

En donantes de sangre de América Latina se ha descrito seropositividad anti-VHE IgG con frecuencias entre 1,2\% y $16,7 \%$, así: $1,8 \%$ y $16,7 \%$ en Argentina $(34,33) ; 16,2 \%$ en Bolivia (35); $2 \%$ a $10 \%$ en Brasil $(9,29,36,37) ; 8 \%$ en Chile (38); $1,4 \%$ en Cuba (39) y $1,2 \%$ en donantes de sangre de Uruguay (40). La frecuencia de $45,2 \%$ encontrada en este estudio es mucho mayor a lo descrito previamente en donantes de sangre de América Latina. Esto podría deberse a que generalmente los donantes de sangre provienen de zonas urbanas, por lo que podrían estar menos expuestos a factores de riesgo para la infección por VHE.

La muestra analizada en el presente estudio no es representativa, además de que el tamaño de muestra es muy limitado y por tanto no se pueden comparar los resultados con otros estudios realizados en poblaciones rurales y de donantes de sangre en América Latina. Con respecto a la técnica utilizada para la detección de anticuerpos anti-VHE, la mayoría de los inmunoensayos comerciales para la detección de IgM e IgG contra el VHE están basados en antígenos sintéticos del VHE derivados de los ORF2 y ORF3, de las cepas de México (genotipo 2) y Myanmar (genotipo 1) (41). Schnegg y colaboradores evaluaron la sensibilidad del kit utilizado en este estudio mediante un panel de 20 sueros, 15 obtenidos de pacientes con infección aguda por VHE y 5 con infección postaguda ( 4 a 14 semanas después de la fase aguda) (42): el $80 \%$ de las muestras en fase aguda y el $20 \%$ de las muestras postagudas fueron positivas para 
IgG, para una sensibilidad total del $65 \%$. Avellón y colaboradores evaluaron la sensibilidad de varios estuches comerciales para la detección de anticuerpos anti-VHE genotipo 3; para el estuche comercial utilizado en el presente estudio (Dia.pro), encontraron una sensibilidad anti-VHE tipo IgM del 59,6\% y anti-VHE tipo IgG del 77,5\% (43). Estos resultados contrastan con la sensibilidad del $99 \%$ reportada por el fabricante. Otros autores no reportan problemas de sensibilidad en la detección del genotipo 3 del VHE (43).

\section{CONCLUSIONES}

Es importante considerar que en todos los estudios que se han realizado hasta el momento en Colombia, se han utilizado estuches comerciales del inmunoensayo de la misma casa comercial (Dia.pro) para la detección de anticuerpos anti-VHE, como en el presente estudio. A pesar del limitado tamaño muestral, el presente trabajo corresponde a la primera descripción de anticuerpos anti-VHE en donantes de sangre en Colombia. Este estudio aporta evidencia de la infección por el VHE en la población del municipio de Yarumal (Antioquia), con una seroprevalencia del 45,2\%. La presencia de actividad de ganadería porcina y el limitado acceso al agua potable antes del 2007 podrían considerarse factores de riesgo para esta población, aunque no se detectaron casos de infección aguda (anti-VHE IgM 0\%).

Los resultados de este estudio aportan al conocimiento de la epidemiología de la infección por VHE en Colombia y en América Latina; sin embargo, al tratarse de un tamaño muestral pequeño, es necesario ampliar el número de muestras en la población para confirmar estos hallazgos e identificar claramente sus factores de riesgo. Además, es necesario realizar estudios en donantes de sangre de otras zonas de Colombia, para contribuir al establecimiento de la importancia clínica y epidemiológica de la infección por VHE.

\section{Agradecimientos}

Instituto Tecnológico Metropolitano. Proyecto de Sostenibilidad, Vicerrectoría de Investigación, Universidad de Antioquia.

\section{Financiación}

Instituto Tecnológico Metropolitano (ITM) y Proyecto de Sostenibilidad, Vicerrectoría de Investigación, Universidad de Antioquia.

\section{Conflicto de intereses}

Los autores declaran no tener conflicto de intereses.

\section{REFERENCIAS}

1. World Health Organization (WHO). Hepatitis E Fact sheet \#280 [Internet]. 2015 [citado el 15 de septiembre de 2015]. Disponible en: http://www.who.int/mediacentre/factsheets/fs280/en

2. Smith DB, Simmonds P, Jameel S, Emerson SU, Harrison TJ, Meng X-J, et al. Consensus proposals for classification of the family Hepeviridae. J Gen Virol. 2014;95(Pt 10):2223-32.

3. Sánchez Partidas DA, Gutiérrez García C del R. Virus de la hepatitis E: Características biológicas y epidemiológicas. Rev Soc Venez Microbiol. 2012;32(1):6-12.

4. Lu L, Li C, Hagedorn CH. Phylogenetic analysis of global hepatitis $\mathrm{E}$ virus sequences: genetic diversity, subtypes and zoonosis. Rev Med Virol. 2006;16(1):5-36.

5. Pérez-Gracia MT, García M, Suay B, Mateos-Lindemann ML. Current Knowledge on Hepatitis E. J Clin Transl Hepatol. 2015;3(2):117-26.

6. Khuroo MS, Khuroo MS. Hepatitis E: an emerging global disease - from discovery towards control and cure. J Viral Hepat. 2016;23(2):68-79.

7. Echevarría J m., González J e., Lewis-Ximenez L 1., dos Santos DRL, Munné M s., Pinto M a., et al. Hepatitis E virus infection in Latin America: A review. J Med Virol. 2013;85(6):1037-45.

8. Yugo DM, Meng X-J. Hepatitis E Virus: Foodborne, Waterborne and Zoonotic Transmission. Int J Environ Res Public Health. 2013;10(10):4507-33.

9. Bortoliero AL, Bonametti AM, Morimoto HK, Matsuo T, Reiche EMV. Seroprevalence for hepatitis E virus (HEV) infection among volunteer blood donors of the Regional Blood Bank of Londrina, State of Paraná , Brazil. Rev Inst Med Trop São Paulo. 2006;48(2):87-92.

10. El Sayed Zaki M, El Razek MMA, El Razek HMA. MaternalFetal Hepatitis E Transmission: Is It Underestimated? J Clin Transl Hepatol. 2014;2(2):117-23.

11. Peláez D, Hoyos MC, Rendón JC, Mantilla C, Ospina MC, Cortés-Mancera $\mathrm{F}$, et al. Infección por el virus de la hepatitis $\mathrm{E}$ en pacientes con diagnóstico clínico de hepatitis viral en Colombia. Biomédica. 2014;34(3):354-65.

12. Rendón J, Hoyos MC, di Filippo D, Cortes-Mancera F, Mantilla C, Velasquez MM, et al. Hepatitis E Virus Genotype 3 in Colombia: Survey in Patients with Clinical Diagnosis of Viral Hepatitis. PLoS ONE. 2016;11(2):e0148417.

13. Betancur CA, Mejía MV, Portillo S. Seroprevalencia de hepatitis E en trabajadores de fincas porcícolas del Valle de Aburrá 2011-2012. Acta Médica Colomb. 2013;38(2):68-70.

14. Gutiérrez-Vergara CC, Rodríguez B, Parra-Suescún J, Correa-Londoño G, López-López L, López-Herrera A, et al. Determinación de anticuerpos totales (IgG/IgM) y específicos ( $\operatorname{IgM})$ para el virus de la hepatitis $\mathrm{E}$ y detección molecular del virus en heces de humanos con o sin exposición ocupacional a porcinos en 10 municipios de Antioquia. Iatreia. $2015 ; 28(3): 248-58$.

15. Gutiérrez Vergara CC, Ospina Vélez DA, Forero Duarte JE, Rodríguez BDJ, Gutiérrez Builes LA, Correa Londoño G, et 
al. Detección serológica y molecular del virus de la Hepatitis $\mathrm{E}$ en cerdos de granjas antioqueñas. Rev CES Med Vet Zootec. 2014;9(2):158-68.

16. Forero JE, Parra JE, López A. Detección del genoma del virus de la hepatitis $\mathrm{E}$ en muestras de heces de cerdos en plantas de beneficio en Antioquia, Colombia. Rev Fac Med Vet Zootec. 2014;61(3):221-7.

17. Gutiérrez-Vergara C, Quintero J, Duarte JF, Suescún JP, López-Herrera A. Detection of hepatitis $\mathrm{E}$ virus genome in pig livers in Antioquia, Colombia. Genet Mol Res GMR. 2015;14(1):2890-9.

18. Báez P, Jaramillo CM, Arismendi L, Rendón JC, CortesMancera A, González MM, et al. Evidencia molecular de virus de la hepatits $\mathrm{E}$ en fuentes de agua en Antioquia. Biomédica. 2015;35(1):40-1.

19. Alcaldía de Yarumal. Información General del Municipio [Internet]. [citado el 15 de septiembre de 2015]. Disponible en: http://www.yarumal.gov.co/alcaldia/presentacion.

20. Departamento Administrativo Nacional de Estadísticas (DANE). Censo General 2005. Perfil Yarumal, Antioquia [Internet]. 2010. Disponible en: http://www.dane.gov.co/files/ censo2005/PERFIL_PDF_CG2005/05887T7T000.PDF.

21. Aguas del Norte Antioqueño. Servicio de Acueducto [Internet]. [citado el 15 de septiembre de 2015]. Disponible en: http://www.yarumal.gov.co/aguas/.

22. DIA.PRO. ELISA : HEV Ab [Internet]. [citado el 14 de septiembre de 2015]. Disponible en: https://www.diapro. it/index.php/products/elisa/hev-ab-detail.

23. 21.Peláez-Carvajal D, Martínez-Vargas D, Escalante-Mora M, Palacios-Vivero M, Contreras-Gómez, Lady. Coinfección del virus de la hepatitis $\mathrm{E}$ con otras hepatitis virales en Colombia y su caracterización genotípica. Biomédica. 2016;36(1).(Nota: falta proporcionar datos).

24. Alvarado-Esquivel C, Sánchez-Anguiano LF, HernándezTinoco J. Seroepidemiology of hepatitis E virus infection in general population in rural durango, México. Hepat Mon. 2014;14(6):e16876.

25. Alvarado-Esquivel C, Sánchez-Anguiano LF, HernándezTinoco J. Seroepidemiology of hepatitis E virus infection in mennonites in Mexico. J Clin Med Res. 2015;7(2):103-8.

26. Alvarado-Esquivel C, Sánchez-Anguiano LF, HernándezTinoco J. Hepatitis E virus exposure in pregnant women in rural Durango, Mexico. Ann Hepatol. 2014;13(5):510-7.

27. Bartoloni A, Bartalesi F, Roselli M, Mantella A, Arce CC, Paradisi F, et al. Prevalence of antibodies against hepatitis $\mathrm{A}$ and $\mathrm{E}$ viruses among rural populations of the Chaco region, south-eastern Bolivia. Trop Med Int Health TM IH. 1999;4(9):596-601.

28. Dell'Amico MC, Cavallo A, Gonzales JL, Bonelli SI, Valda Y, Pieri A, et al. Hepatitis E Virus Genotype 3 in Humans and Swine, Bolivia. Emerg Infect Dis. 2011;17(8):1488-90.

29. Trinta K, Liberto M. Hepatitis E virus infection in selected Brazilian populations. Mem Inst Oswaldo Cruz. 2001;96(1):25-9.

30. Vitral CL, da Silva-Nunes M, Pinto MA, de Oliveira JM, Gaspar AMC, Pereira RCC, et al. Hepatitis A and E sero- prevalence and associated risk factors: a community-based cross-sectional survey in rural Amazonia. BMC Infect Dis. 2014;14:458.

31. Silva SMT da, Oliveira JM de, Vitral CL, Vieira K de A, Pinto MA, Souto FJD. Prevalence of hepatitis E virus antibodies in individuals exposed to swine in Mato Grosso, Brazil. Mem Inst Oswaldo Cruz. 2012;107(3):338-41.

32. Pujol FH, Favorov MO, Marcano T, Esté JA, Magris M, Liprandi F, et al. Prevalence of antibodies against hepatitis $\mathrm{E}$ virus among urban and rural populations in Venezuela. J Med Virol. 1994;42(3):234-6.

33. H. Fainboim, J. González, E. Fassio, A. Martínez, L. Otegui, M. Eposto, P. Cahn RM, G. Landeira, G. Suaya, E. Gancedo, $1 \mathrm{R}$. Castro LB and HL. Prevalence of hepatitis viruses in an anti-human immunodeficiency virus-positive population from Argentina. A multicentre study.J Viral Hepat 1. 1999;6(1):53-7.

34. Munné MS, Altabert NR, Otegui M LO, Vladimirsky SN, Moreiro R, Espul MP, et al. Updating the knowledge of hepatitis E: new variants and higher prevalence of anti-HEV in Argentina. Ann Hepatol. 2014;13(5):496-502.

35. Konomi N, Miyoshi C, La Fuente Zerain C, Li T-C, Arakawa Y, Abe K. Epidemiology of Hepatitis B, C, E, and G Virus Infections and Molecular Analysis of Hepatitis G Virus Isolates in Bolivia. J Clin Microbiol. 1999;37(10):3291-5.

36. Parana R, Cotrim HP, Cortey-Boennec ML, Trepo C, Lyra L. Prevalence of hepatitis $\mathrm{E}$ virus IgG antibodies in patients from a referral unit of liver diseases in Salvador, Bahia, Brazil. Am J Trop Med Hyg. 1997;57(1):60-1.

37. Passos-Castilho AM, de Sena A, Geraldo A, Spada C, Granato CFH. High prevalence of hepatitis E virus antibodies among blood donors in Southern Brazil. J Med Virol. 2016;88(2):361-4

38. Ibarra H, Riedemann S, Reinhardt G, Frieck P, Siegel F, Toledo C, et al. [Prevalence of hepatitis E virus antibodies in blood donors and other population groups in southern Chile]. Rev Médica Chile. 1997;125(3):275-8.

39. Lemos G, Jameel S, Panda S, Rivera L, Rodríguez L, Gavilondo JV. Hepatitis E virus in Cuba. J Clin Virol Off Publ Pan Am Soc Clin Virol. 2000;16(1):71-5.

40. Cruells MR, Mescia G, Gaibisso R, Ramírez M, Gutiérrez M, Kohen S, et al. [Epidemiological study of hepatitis A and $\mathrm{E}$ viruses in different populations in Uruguay]. Gastroenterol Hepatol. 1997;20(6):295-8.

41. Pas SD, Streefkerk RHRA, Pronk M, de Man RA, Beersma $\mathrm{MF}$, Osterhaus ADME, et al. Diagnostic performance of selected commercial HEV IgM and IgG ELISAs for immunocompromised and immunocompetent patients. J Clin Virol Off Publ Pan Am Soc Clin Virol. 2013;58(4):629-34.

42. Schnegg A, Bürgisser P, André C, Kenfak-Foguena A, Canellini G, Moradpour D, et al. An Analysis of the Benefit of Using HEV Genotype 3 Antigens in Detecting Anti-HEV IgG in a European Population. PLoS ONE. 2013;8(5):e62980

43. Avellon A, Morago L, García-Galera del Carmen M, Muñoz M, Echevarría J-M. Comparative sensitivity of commercial tests for hepatitis E genotype 3 virus antibody detection. J Med Virol. 2015;87(11):1934-9. 